\title{
Connecting strategies on dried blood spots
}

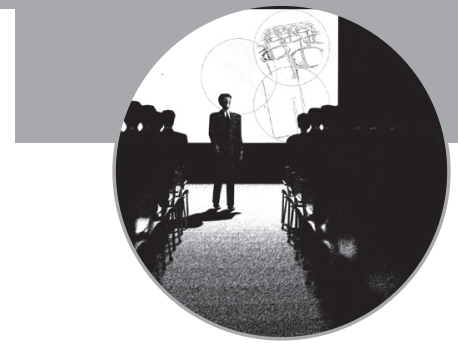

\section{European Bioanalysis Forum Workshop: Connecting strategies on dried blood spots}

Sheraton Hotel, Brussels, Belgium, 17-18 June 2010

The European Bioanalysis Forum is a non-profit organization comprised of European pharmaceutical companies (25 members to date). Their activities focus on bringing together managers and scientists in the broad field of bioanalysis to discuss topics related to science, process and regulations. There has been much interest over the past few years in the potential application of dried blood spots as an alternative to traditional plasma collection in pharmacokinetic studies. The success of the technique has been highlighted by several companies. We know that seven of the European Bioanalysis Forum member companies are using dried blood spots intensively and that 22 out of 25 companies are using it or plan to use it very soon, initially in nonregulated studies. However, most companies have less than I year of experience with dried blood spots and, beyond the scientific merit, it is less clear just how the technique is perceived by key client groups, such as toxicology, clinical and regulatory authorities. The objective of this symposium was to bring together representatives from all of these client groups as well as a broad bioanalytical audience to discuss the various perspectives on dried blood spots and to try to provide answers to potential issues that still remain. The symposium included sessions on dried blood spots in the nonregulated environment, toxicology and regulatory/QA perspectives, clinical use and bioanalytical applications and tools. There was plenty of time for discussion within the program in tutorials, poster and break-out sessions and the degree of delegate participation reflected the high level of engagement with the topic. A total of 190 delegates attended from more than 80 organizations. In addition to 21 oral presentations, more than 20 posters were presented and there was a vendor exposition of ten sponsor companies.

In the introductory talk, Philip Timmerman (Johnson \& Johnson, Belgium), presented on behalf of the European Bioanalysis Forum (EBF) on the current status of the technique. In his presentation, Philip gave feedback from two surveys held within the EBF. The first one touched on the current level of involvement of EBF companies with dried blood spots (DBS) and their plans to further develop the technique within their companies. Some highlights are presented in this article. In the second survey, EBF looked at what parameters might need to be changed with respect to validation of bioanalytical methods when applied to DBS methods. The results of this survey will form the basis of continued discussions EBF intends to have within the bioanalytical community, and will lead to a recommendation from the EBF on validation and application of DBS methods in clinical and preclinical studies.

\section{DBS in the nonregulated environment}

Kevin Beaumont (Pfizer) initiated the nonregulatory discussions during his presentation 'Who decided we should measure plasma?' where he made the case for blood analysis by DBS as pharmacokinetic parameters are generally based on blood rather than plasma. Beaumont described the use of the Well Stirred Model, where clearance is related back to hepatic blood flow, and how for CNS compounds, distribution across the blood-brain barrier is vital for target penetration. Examples were presented whereby, owing to differences in blood-to-plasma ratio, some different conclusions can be drawn depending on which matrix is selected. Examples for both basic and acidic compounds were presented, where it was clear that a different conclusion would have been made when pharmacokinetic parameters were calculated using blood concentrations or plasma concentrations.

Frank Picard provided a comprehensive overview of how DBS has been evaluated and utilized at Novartis. The introduction opened with a review of how pharmaceutical companies have been assessing DBS followed by specific work performed at Novartis. In total, 13 different compounds have been evaluated mostly within nonregulated studies; however, data were generated from two compounds in preclinical and clinical development studies. Procedures were outlined from sample collection by finger prick
Richard Abbott', John Smeraglia ${ }^{2}$, Stephen White ${ }^{3}$, Silke Luedtke ${ }^{4}$, Leonarda Brunet ${ }^{5}$, Elizabeth Thomas', Suzanne Globig ${ }^{7}$ \& Philip Timmerman ${ }^{+8}$

'Shire Pharmaceuticals, Basingstoke, UK

2Pfizer, Sandwich, UK ${ }^{3}$ GlaxoSmithKline, Ware, UK ${ }^{4}$ Boehringher-Ingelheim, Biberach, Germany

${ }^{5}$ Abbott, Daix, France

${ }^{6}$ AstraZeneca, Alderley Park, UK ${ }^{7}$ Actelion, Basel, Switzerland

${ }^{8}$ Bioanalysis Department, Johnson \& Johnson Pharmaceutical Research \& Development, a Division of Janssen Pharmaceutica NV, Turnhoutseweg 30, 2340 Beerse, Belgium

${ }^{\dagger}$ Author for correspondence:

E-mail: ptimmerm@prdbe.jnj.com

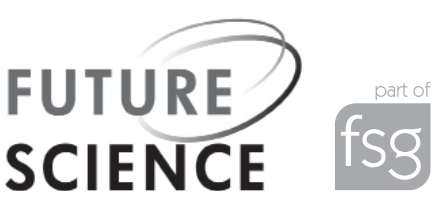


in humans to ocular bleeds in rats on treated and nontreated paper and development of methodologies for quantification, including measurement using chip technology. The overall assessment included parameters such as type of card selected, spot homogeneity, hematocrit, blood volume and drying time as well as standard method assessments such as sensitivity and selectively. Significantly, DBS sampling was compared with conventional sampling techniques. Data were also presented that showed that for certain unstable compounds it is more challenging to use DBS as it can be more difficult to incorporate enzyme inhibitors at the point of collection. However, advantages such as cost savings, the convenience of sample collection in a less invasive manner and reduced blood collection, as well as the reliability of quantitative data, has led to an increased use of the technique at Novartis. Other considerations must be taken into account, such as the reliability of spotting techniques, difficulties in achieving a picogram level lower limit of quantification (LLOQ) and the impact of hematocrit. However, DBS is viewed as a promising, convenient approach that can replace conventional plasma collection.

Patricia Wright (Pfizer) provided a perspective on the use of DBS for metabolite identification and shared data on experiments performed to determine the feasibility of using DBS samples to characterize metabolism. She began with the requirements and impact of assessing metabolism in in vitro and in vivo systems, which included an outline of conventional procedures using plasma. The current procedure of using residual plasma samples following pharmacokinetic (PK) assessment was described. Samples are pooled in a time-normalized manner (e.g., $1 \mathrm{~h}-10 \mu \mathrm{l}, 2 \mathrm{~h}$ $-10 \mu \mathrm{l}, 5 \mathrm{~h}-30 \mu \mathrm{l}, 10 \mathrm{~h}-50 \mu \mathrm{l}$ [total $100 \mu \mathrm{l}])$ followed by protein precipitation and analysis by LC-MS using high-resolution instrumentation. The feasibility of performing metabolite identification from DBS samples was assessed by spiking four separate compounds and their related metabolites over a wide range of chemical space into whole blood and plasma. Liquid whole blood, DBS and plasma-spiked samples were then analyzed and gave comparable results. It was noted that while assessment of metabolites in spiked samples was feasible, pooling of DBS samples in a time-normalized manner is not without its challenges owing to the limited amount of residual sample after PK analysis and the availability of limited punch diameters. It is also difficult to determine the risk of metabolites sticking to paper, which would only be apparent once the definitive radiolabeled studies are performed. The conclusion was that performing metabolite identification by DBS is feasible, however greater experimentation is required to characterize metabolism using DBS samples.

Christopher Smith (AstraZeneca) presented on metabolomic analysis of dried biofluids including plasma, blood and urine. The data presented were from biofluids collected on untreated paper where the total ion chromatogram was collected from DBS, dried plasma samples (DPS) and protein precipitated plasma samples in both negative and positive ionization mode. Reliable coefficients of variation were obtained from DBS and DPS samples, however it was noted that phospholipids were retained on the paper. Dried urine samples were also assessed for their metabolomic profile and also provided good CVs when compared with liquid urine. The preliminary data indicated that collection of dried samples can provide an alternative approach to metabolomics, especially where sample volume is a concern; however, there are limitations on the achievable sensitivity, which is driven by the low volume used. Alternative tools, such as nanospray and chip-based analysis, can be helpful, however improvements to achieve greater sensitivity are still required.

\section{Combined toxicology/regulatoryl QA session}

Fiona McClure (GlaxoSmithKline [GSK]) described in her presentation 'Spot the difference - effect of toxicology (TK) blood sampling on clinical pathology parameters' an example where hematology samples were taken from the TK group animals (on day 4) of a 1-month rat study. As expected there was a drop in hemoglobin levels but an increase in total reticulocyte count (more pronounced in the females), due to the TK bleed volume from these animals. McClure went on to explain that reticulocytes can be classified into three types depending on their maturity. By use of staining techniques it is possible to use the early reticulocytes as an early indicator of what is happening in the bone marrow following a bleed. It is important to note that any observed changes in hemoglobin and/or reticulocyte counts are also observed in control group animals. Fiona then described a rat DBS study designed to investigate whether taking TK bleeds from main toxicology group animals would impact on standard toxicology end points. While hemoglobin and total reticulocyte counts showed no significant effect, it was possible to see an effect when monitoring the reticulocyte 
subpopulations. However, these changes were mirrored in the control group animals and could be minimized by reducing the blood volume taken per time point to $40 \mu$ l. In concluding, McClure stated that small-volume TK samples can be taken from main toxicology group animals without affecting standard parameters. This can facilitate the removal of satellite animals, which is an approach now adopted by GSK for all non-good laboratory practice (GLP) rat TK studies.

Laura Patrone (BMS) presented 'Direct comparison of DBS analysis to plasma and whole blood analysis in toxicokinetic studies of rats'. Patrone described the various goals and end points of blood collection in in vivo toxicokinetic (TK) studies and highlighted that the typical requirements for large blood volumes can be problematic for rodents and for mice in particular. A pilot study was conducted at BMS to compare TK data from whole blood, DBS and plasma. Two tool compounds were tested (compound $\mathrm{X}$ and omeprazole), and plasma data were converted into predicted blood concentrations using the appropriate blood:plasma ratio. For both compounds the TK data presented showed good correlation between DBS, whole blood and predicted blood concentrations. It was noted that there was some variation between blood and DBS results for omeprazole at low concentrations, and that whole blood data were slightly more variable, which could be attributed to difficulties in handling this matrix. It was also noted that incurred sample reproducibility (ISR) conducted on DBS samples showed good agreement with the original analysis. In conclusion, Patrone explained that using DBS in combination with microsampling can offer several advantages during sample collection, processing and shipping, but assay development may be more difficult for hydrophilic compounds. She also drew attention to the fact that storage of cards during drying needs consideration, as well as more automated approaches for the future.

Josephine Burnett (Covance) presented the 'Practical application of dried blood spot techniques in toxicology'. Burnett described their procedures for DBS sampling for rats, mice and rabbits, highlighting the fact that animal discomfort is minimal and that if a needle blocks preventing blood draw, then a blood spot can be acquired from the puncture site, thus removing the need for multiple punctures. Burnett described the benefits of using DBS, in terms of the $3 R s$, reductions in test compound requirements, sample processing, shipping costs and other key benefits. The process flows from bleed to analysis were compared for traditional plasma analysis and DBS, highlighting a reduction in the number of steps from 13 to six. Burnett shared positive feedback from animal technicians and sample management colleagues and then described proposed study designs for rats and mice using DBS. Final remarks included consideration that the bioanalytical method must be in place well in advance of sampling in order for the card type to be specified.

Marcus Benton (Fulcrum Pharma, on behalf of BARQA) gave a ' $\mathrm{QA}$ perspective on $\mathrm{DBS}$ analysis'. Marcus listed the various international standards and norms that may need to be considered, but pointed out that in general terms the requirements are very similar. Attention was drawn to some specifics of GCLP, ISO 17025 , and the guidance for industry reference paper for bioanalytical method validation [1]. For DBS methods the usual validation criteria apply, with the possible exception of freeze-thaw, but other parameters may need to be considered, such as analyte and metabolite stability on card, volume of blood spotted, device used for spotting and temperature of blood spotted. Marcus raised the question whether or not using DBS provides the same QA/QC challenges as any other clinical pathology or drug level analysis. He went on to highlight considerations around training of nonmedical personnel for DBS sampling and the possible effect of blood spot diameter, hematocrit value and age of blood used for calibrator and QC preparation. Concluding remarks included discussion of the possible need for method transfers, cost-benefit considerations and acquisition of metrics (e.g., number of unacceptable results and normal values for hemoglobin/hematocrit).

\section{Clinical session}

Achiel Van Peer (Johnson \& Johnson) in his presentation 'Blood and plasma: a magic twin or single in human pharmacokinetics?' gave his personal thoughts on using DBS as an alternative to plasma for human PK considerations. Historically, plasma is commonly used as the main matrix to measure systemic drug concentrations for 'practical reasons', such as lack of cellular material and the ease of manipulation and extraction. However, based on physiological considerations whole blood is more relevant with respect to PK: hepatic clearance is expressed in terms of blood flow and unbound drug blood concentration. van Peer listed the most recent regulatory EMA guidelines [2,3] and draft US FDA Guidances [101,102] and pointed out 
that there is no consensus about plasma or blood concentration-time profiles. However, to move forward with DBS, the fundamental question as described in the recent commentary by Malcolm Rowland and Gary T Emmons [4] is, "When blood or plasma ceases to be an appropriate surrogate, what steps need to be taken to make the necessary correction?". Measuring PK in plasma, we make the fundamental assumption that the compound partitions evenly between plasma and red blood cells. For many compounds, this is the case and consequently PK in plasma reflects the PK in blood. However, van Peer presented several examples that clearly illustrated the importance of the magnitude of the whole blood to plasma drug concentration ratio when interpreting concentration-time profiles and derived PK parameters. Constancy in fraction unbound for plasma and blood, constancy of hematocrit, blood cell affinity of the compound and concentration dependency of protein binding are factors to investigate with early in vitro assessments using blood from the study population of interest prior to implementing the use of DBS in a drug development program.

Hitesh Pandya (University of Leicester) presented the Caffeine Using Blood Spots (CUBS) study, which is a proof-of-concept study designed by the collaboration between University of Leicester and GSK to answer the following question: 'PK studies in infants and children: are dried blood spots the answer?'. Most of the drugs currently used to treat the pediatric population have not been studied or authorized for use and dose regimens are based on PK data generated in adults. This practice is unsafe and no longer acceptable to regulatory authorities as there are applicable regulations and guidelines that create obligations with regard to conducting clinical trials in pediatric patients, including neonates (Regulation [EC] 1901/2006, EMA Guideline/536810/2008). PK studies in the pediatric population encompass multiple ethical and technical difficulties linked to age, such as relatively large volumes of blood and repeated venipuncture blood collection, which remain major obstacles to parents and ethics committees. To test whether DBS sampling can overcome blood sampling issues in children, the CUBS study group designed a proof-of-principle study to determine caffeine PK in preterm infants. The preliminary conclusions of this study are very positive and encouraging. The recruitment of preterm patients is above expectations, parents and staff find the DBS method easy and acceptable. However, it should be noted that sampling issues were identified in intensive care. The number of spots/ cards (ten DBS spots) and total blood volume $(0.6 \mathrm{ml})$ per patient using DBS blood is comfortably within the volume limits for sampling recommended in the EMA guideline. Training and monitoring for DBS sampling and methods is of great importance. Pandya concluded that further 'in field' studies and strong partnerships between clinicians, academia and industry are needed and are essential to establish the DBS technique for general use.

Leo Stolk (University Hospital of Maastricht, The Netherlands) started his presentation 'Dried blood spot methods in therapeutic drug monitoring (TDM): methods, assays and pitfalls' with a review of the TDM assays based on DBS sampling described in the literature since the 1980s and especially during the last few years (>40 papers). This review was recently published by the scientific committee for new sampling strategies of the International Association for Therapeutic Drug Monitoring and Clinical Toxicology [5]. The analytical and sampling techniques involved and factors that may influence the accuracy and reproducibility of DBS methods in TDM were discussed. If a wide range of analytical techniques were used with DBS, sensitive and specific methods with tandem MS detection seem preferable. Both analytical and clinical validations were generally covered, with some exceptions for comparison of concentrations in venous samples and DBS samples collected at the same time. Stability was generally investigated and in most cases analytes were reported as stable. It was noted that stability of DBS cards in the whole range of temperature conditions that could be expected during shipment was rarely examined. With respect to blood sampling methods, the numerous advantages of DBS in TDM were discussed, such as blood collection and spotting by the patient himself at home, which enable ambient conditions, storage at ambient temperature and shipment via normal postal systems with no special biohazard arrangements. Stolk pointed out that the DBS sampling by patients themselves is not under control and critical aspects may need to be considered, such as blood spotting using a pipette, handling and drying. Sharing his own experience, he highlighted that there should be adequate instructions and training as well as quality control of the DBS cards on their arrival at the laboratory. Influence of blood properties (hematocrit values for patients' samples, use of hemolyzed blood or deviating hematocrit for preparation of 
calibrators), extraction methods and risk of contamination are other parameters that should also be addressed. In conclusion, DBS appears to be a good alternative to conventional venous sampling and a good approach for TDM. However, for each application, benefit should be weighed against potential errors that could be introduced with DBS. There is evidently a need for more standardization and automation, basic research and assay development.

\section{Bioanalytical applications}

Paul Abu-Rabie (GSK) described 'Direct quantitative bioanalysis of drugs in dried blood spot samples' in his presentation. He pointed out that the ethical, financial and organizational advantages of the application of DBS within the development process are well recognized. However, for the bioanalyst, DBS is challenging because switching from plasma to blood adds more complexity and requires a change to wellestablished method validation and sample analysis procedures. To move DBS forward from a bioanalytical point of view with a focus on automated, high-throughput analysis the direct analysis of DBS samples would be preferred over the currently established semi-automated extraction procedure. Direct analysis can be achieved either by various direct desorption techniques such as direct analysis in real time, desorption electrospray, laser electrospray ionization, paper spray analysis or by direct elution. GSK in particular is working with various instrument vendors to evaluate the most suitable interface that will allow the bioanalyst to work according to his/her established plasma standards with respect to assay throughput, linearity and sensitivity.

Hesham Ghobarah (AB Sciex) described the challenges of performing DBS bioanalysis on inhalation drugs with low systemic circulation in his presentation 'High sensitivity LC-MS/MS quantification of corticosteroids in dried blood spots and Evaluation of Software Saturation correction for extending dynamic range'. Inhalation drugs usually require a LLOQ in the low $\mathrm{pg} / \mathrm{ml}$ range, which cannot be achieved using DBS and a common LC-MS/MS system. Another challenge of inhalation drugs, such as budesonide. Is the need for a rather wide dynamic range due to high $\mathrm{C}_{\max }$ values but short half-life of the analyte. To cope with this requirement, $\mathrm{AB}$ Sciex developed a new integration algorithm entitled Signal Finder ${ }^{\mathrm{TM}}$, which corrects saturation effects at the upper limit of quantification (ULOQ) and therefore allows a wider linear range. By increasing the linear range the need for sample dilution is reduced, and dilution integrity issues discovered with DBS can be overcome. The required sensitivity can be achieved using a UPLC system coupled to a high-sensitivity triple quadrupole mass spectrometer.

Matthew Barfield (GSK, 'The use of dried plasma spots [DPS] and dried urine spots [DUS] for LC-MS/MS assays') reported on the experiences at GSK with respect to method validation and study sample analysis. To date, 231 DBS methods have been validated for 105 compounds. A total of 168 nonclinical GLP studies were performed using DBS cards (20357 samples and 85 different analytes analyzed in these studies), 86 nonclinical GLP studies (18829 samples and 35 different analytes analyzed in these studies) and nine clinical studies (4027 samples and 15 different analytes analyzed in these studies). He also touched on the economical advantage of DBS. GSK estimated cost savings of GBP£5-8 million a year if all toxicokinetic and clinical studies were performed using DBS instead of 'conventional' plasma samples. The cost benefit offered by the card technology could also be used for DPS and DUS because feasibility studies performed at GSK showed that plasma and urine may also be spotted on the filter cards. DPS may therefore be an attractive option for compounds already in a later phase of development where a switch in matrix would not be sensible.

Almudena Gajate Perez (Roche) discussed 'Can DBS stabilize ester prodrugs and glucuronide metabolites?' Four labile compounds that require the addition of inhibitors as well as cooled storage/processing in conventional plasma analysis were chosen as model compounds. Two compounds were ester prodrugs, one was a glucuronide and the last was known to hydrolyze rather easily. The outcome of the feasibility study was that the use of DBS samples instead of plasma or whole blood samples generally improved the stability of the model compounds in the short term. Long-term stability was compound dependent. Generally, the stability was strongly dependent on the type of card used for the experiments. Fast drying as well as storage at low temperatures seemed to be beneficial with respect to stability enhancement. Gajate Perez also concluded that the coating of treated cards may stop enzymatic activity but may not stop chemical degradation.

Martyn Hemsley (Covance) described the logistical advantages of DBS in his presentation entitled 'Logistical considerations and experiences in utilizing dried blood spots in 
quantitative bioanalysis'. The Guidelines for the Shipment of Dried Blood Spot Specimens released by the Centers for Disease Control and Prevention (USA), state that dried spots can be transported via normal postal systems without special mailing cartons. The cost savings due to shipment at room temperature and even the possibility to ship via normal postal systems instead of by courier are obvious. However, during shipment each card should be stored individually in a sealable, polypropylene bag with a desiccant pouch because the presence of moisture can cause problems later during the extraction process and may also jeopardize the stability of the analyte. The handling of DBS cards is less hazardous than plasma because the cards offer bacterial lysis and viral inactivation, according to the providers.

\section{Tools for bioanalysis of DBS: tools}

Bert Ooms (Spark Holland) presented on the subject of 'Online DBS-SPE-MS/MS - feasibility of a concept for rapid DBS analysis without punching. For DBS-SPE-MS/MS, both online DBS extraction with a loop interface and online DBS extraction with a SPE interface were investigated. In addition, Spark also looked at whole blood online SPE, using a high-pressure solvent dispenser. The extraction method was described - an exact volume of blood can be applied onto a sorbent sampling cartridge. IS can be applied on top of this. This is then eluted onto a SPE cartridge which is then eluted onto the LC-MS/MS system. Working at GSK, the DBS cards were clamped into the system, a stream of liquid flowed through the spot onto the SPE cartridge and from there onto the LC-MS/MS system. DBS cards containing IS were eluted onto the system. DBS cards were also eluted with a solution of IS onto the system. At Spark Holland, the DBS cards were clamped into the system, a stream of liquid flowed through the spot onto the 8- $\mu \mathrm{m}$ C18 SPE cartridge and from there into the MS system (no column). Whatman protein saver 903 cards were used. In total, $15 \mu$ of blood was spotted onto the card. A sample containing a mixture of propranolol, haloperidol, amitriptyline and verapamil was examined. Gradient elution was used. Good separation and good precision data were observed. Samples were analyzed at a rate of 20 samples/h and the assay was linear in the range 1 to $1000 \mathrm{ng} / \mathrm{ml}$.

So what's next? Spark have a new device at the concept stage with a fully automated card selection from a stacker, ID, inspection and online extraction.
Gary Harland (Waters) described 'The use of an integrated microfluidic LC-MS/MS device for DBS assays, an approach to increased sensitivity'. Current bottlenecks were highlighted - sample processing, method development, limited sample volume and sensitivity (especially for inhaled and biopharmaceutical products).

Solutions for small sample volume issues were proposed - more sensitive detector, sample enrichment, capillary and nanoscale chromatography. When using capillary and nano-LC we can elute in smaller volumes; increase in sensitivity is inversely proportional to the square of column radius for equal sample load.

TRIZAIC ${ }^{\mathrm{TM}}$ was described. This is a microfabricated ceramic tile, which can be a UPLC or HPLC device (1.7- $\mu \mathrm{m}$ particles). The nano Aquity system can be used to inject on to the TRIZAIC. The TRIZAIC has low system volumes with decreased band broadening and higher sensitivity. The performance is similar to a capillary column. Waters have seen gains of sensitivity when moving from UPLC to TRIZAIC.

The Xevo TQS was also reviewed. This instrument has a new, off-axis ion source technology, known as StepWave ${ }^{\mathrm{TM}}$, which has greater sensitivity than the Xevo ${ }^{\mathrm{TM}} \mathrm{TQ}$. It also features ScanWave collision cell technology to provide enhanced spectral LC-MS/MS data acquisition capabilities, and Radar - an information-rich acquisition approach that allows the collection of highly specific quantitative data for target compounds while providing the ability to visualize all other components

Waters coupled the TRIZAIC and the Xevo TQS, and compared compounds run with UPLC on an API 5000. Silamiquine and extendin (large molecule) both showed large gains in sensitivity. Analysis times as low as $3 \mathrm{~min}$ are possible.

Waters will be launching a DBS website in the near future [103]. This site is independent of Waters and will be a site where people in the DBS community can share knowledge and best practice.

John Dinan (BSD Robotics) presented on 'BSD robotics: bringing the DBS advantage into the laboratory'. He introduced the advantages and applications of 226 paper/cards. John also introduced the audience to the robotic units BSD 600, BSD 600 (Duet) and BAD 700. These are much faster than manual handling and allow positive identification of the punches in the plate wells. They have a versatile dual punch system, a series 3 quick-release punch, and utilize a barcode reader and plate maps with easy-to-use software. Finally, Dinan described 
the BSD 1000 - a unit with minimal operator involvement, positive identification of the spot and integration with other systems. Storage of three magazines is possible with 100 cassetted cards/magazine. The tray carousel has a capacity for six plates. The system automatically scans cards and determines the best position to punch.

The following are available as accessories or may be standard depending in the instrument model: low-pressure air system, light-targeting system, computer controlled, disk detection, dust extraction, autotrigger, humidifier, barcode reader and multistrike function.

BSD is working with Watson LIMS to ensure compatibility.

\section{Summary of breakout sessions}

Below is listed a summary of the main discussion points from each Workshop session.

\section{- Discovery}

Discussion points included:

- The value of using DBS in discovery;

- Importance of training of personnel;

- Blood sampling in small animals, (processing of samples: number of samples; automation and storage) and PK/PD;

- Need for long-term stability for DBS;

- Stability of metabolites/prodrugs; impact of different filter papers;

- Conclusion: blood is a valuable matrix for drug measurement.

\section{- Toxicology}

Discussion points included:

- Removal of satellite animals in GLP studies;

- The influence of different strains (Wistar rat, Sprague Dawley rat) due to size and the impact on blood sampling;

- Composite profiles in mice;

- Awareness of the use of DBS by regulation/authorities;

- Use of DBS for metabolite identification.

\section{Clinic}

Discussion points included:

- Importance of training of personnel (similarity to current training practices for non-DBS, use of training videos, pre-study initiation);

- Use of DBS in pediatrics: great acceptance from hospital nurse and parents (not invasive, reduced volume);
- Differences/opportunities for the use of DBS in global drug development;

- Difference between accurate drug concentrations (could not be performed at home) versus therapeutic drug monitoring: provides a concentration window or defined concentration threshold (could be done at home);

- Discussion on alternatives to DBS: capillary blood can be treated to receive homogenous blood fluid;

- Importance of connecting people: hospital staff, bioanalytical staff and pharmaceutical industry. Pharma should inform, communicate and interact with the hospital staff for expected therapeutic window if switch from plasma to blood concentration.

\section{- Bioanalysis}

Discussion points included:

- Different approaches of addition of the ISTD: to the punched disc;

- Comparison capillary - venous blood and impact on validation?

- Extraction recovery: comparison of approaches such as the 'Covance method' (correction factor that includes the diameters) whole punch;

- Stability of blood: how long can blood be stored prior to spotting? Can frozen blood be an alternative?

- Automation;

- ISR (GSK successfully performed 34 ISR);

- Investigation on humidity and temperature.

\section{Final comment}

The aim of the workshop was to connect people of different disciplines and this was successfully achieved. The conference was not dominated by bioanalytical staff topics. The networks built here should be utilized in the future and the conference will be a starting point for EBF to work on the challenges in bioanalysis and to bring forward recommendations for the use of DBS in the bioanalytical community in the near future.

Financial \& competing interests disclosure The authors have no relevant affliations or financial involvement with any organization or entity with a financial interest in or financial conflict with the subject matter or materials discussed in the manuscript. This includes employment, consultancies, honoraria, stock ownership or options, expert testimony, grants or patents received or pending, or royalties. No writing assistance was utilized in the production of this manuscript. 


\section{News \& ANalysis | CONFERENCE Report}

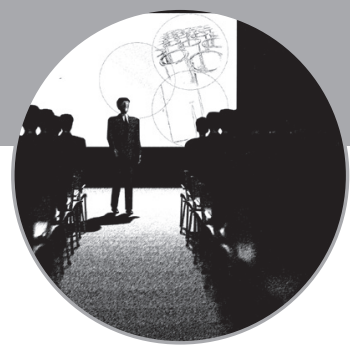

\section{Bibliography}

1 Guidance for Industry Bioanalytical Method Validation. US Department of Health and Human Services Food and Drug Administration. Center for Drug Evaluation and Research (CDER), Center for Veterinary Medicine (CVM), May (2001).

2 EMA. Guideline on the Investigation of Bioequivalence. CPMP/AWP/EWP/1401/98 Rev.1 20 January 2010.

3 EMA. Draft guideline on the investigation of drug interactions. CPMP/EWP/560/95/Rev. 1 April 2010.

4 Rowland M, Emmons GT. Use of dried blood spots in drug development: pharmacokinetic considerations. AAPS J. 12(3), 290-293 (2010).

5 Edelbroek PM, van der Heijden J, Stolk LM. Dried blood spot methods in therapeutic drug monitoring: methods, assays, and pitfalls. Ther. Drug Monit. 31(3), 327-336 (2009).

\section{- Websites}

101 US FDA draft guidance on the content of the Clinical Pharmacology Section of Labeling. www.fda.gov/downloads/drugs/ GuidanceComplianceRegulatoryInformation/ Guidances/ucm109739.pdf

102 US FDA draft guidance on pharmacokinetics in patients with impaired renal function. www.fda.gov/downloads/Drugs/ GuidanceComplianceRegulatoryInformation/ Guidances/UCM204959.pdf

103 Waters.

www.waters.com/bloodspots 